\title{
Reliability of Concentric, Eccentric and Isometric Knee Extension and Flexion when using the REV9000 Isokinetic Dynamometer
}

\author{
by \\ Alberto César Pereira de Carvalho Froufe Andrade 1,2, Paolo Caserotti ${ }^{4}$, \\ Carlos Manuel Pereira de Carvalho 1,2, Eduardo André de Azevedo Abade 1,3, \\ António Jaime da Eira Sampaio ${ }^{1,3}$
}

The aim of this study was to assess the reliability of isokinetic and ISO knee extensor and flexor muscle strength when using the REV9000 (Technogym) isokinetic dynamometer. Moreover, the reliability of several strength imbalance indices and bilateral ratios were also examined. Twenty-four physically active healthy subjects (age $23 \pm 3$ years) underwent three testing sessions, two on the same day and a third, 7 days later. All sessions proceeded in the same order: five concentric contractions at $60^{\circ} \mathrm{s}-1$ followed by an isometric contraction (5 seconds) and five eccentric contractions $\left(60^{\circ} s-1\right)$. The results of this study showed a high reproducibility in eccentric (0.95-0.97), concentric (0.950.96) and isometric (0.93-0.96), isokinetic strength for knee extensor and flexor muscles, thus indicating that the REV9000 isokinetic dynamometer can be used in future sports performance studies. A low-to-moderate reliability was found in the isokinetic strength bilateral ratios while the Hamstring:Quadricep concentric ratio showed moderate reliability. The highest reliability (>0.90) was observed in the dynamic control ratio (Hamstring eccentric:Quadricep concentric) which consequently confirms that it is a more valid indicator for imbalanced reciprocal parameters and can be used in rehabilitation and sports medicine.

Key words: reproducibility, quadriceps, hamstring, peak torque, imbalance ratios, isokinetics.

\section{Introduction}

The use of isokinetic dynamometers to assess muscle function has become progressively popular in sport, research and clinical settings. Isokinetic devices assess joint and muscle maximal concentric $(\mathrm{CON})$, eccentric (ECC) and isometric (ISO) strength under constant velocities throughout the whole range of motion. Several studies have used isokinetic dynamometers to assess ISO and dynamic (CON and ECC) strength of the knee extensor and flexor muscles (Bardis et al., 2004; Cotte and Ferret, 2003). Isokinetic devices are not only used in rehabilitation and sports medicine to assess risk factors such as low strength muscles but also in developing rehabilitation programmes for knee and hamstring injuries or imbalances (Croisier, 2004; Dauty et al., 2003; Impellizzeri et al., 2008; Kannus, 1994). Additionally, some authors have suggested the use of preseason screening of unilateral and bilateral strength imbalance in healthy subjects so as to identify the athletes at a high risk of incurring lower limb injuries during training or competition (Croisier, 2004). It seems consensual that reliable measures are essential to improve the accuracy of ISO, ECC and CON strength evaluations. There are numerous factors that can influence the measurement of

\footnotetext{
1 - Research Center of Sports Science, Health and Human Development (CIDESD).

2 - High Institute of Maia (ISMAI), Portugal.

3 - University of Trás-os-Montes e Alto Douro (UTAD), Portugal.

4 - University of Southern Denmark, Institute of Sports Science and Clinical Biomechanics.
} 
reproducibility, such as measuring devices, testing procedures and the individuals' unique characteristics.

Some studies have previously evaluated the reliability of isokinetic devices such as Biodex (Feiring et al., 1990; Lund et al., 2005; Ordway et al., 2006), Cybex (Bandy and Mclaughlin, 1993; Blacker et al., 2010; Impellizzeri et al., 2008; Li et al., 1996), Kin Com (Sole et al., 2007; Tredinnick and Duncan, 1988), Con-Trex (Bardis et al., 2004; Maffiuletti et al., 2007), Merac (Capranica et al., 1998), Lido (Iga et al., 2006) and iSAM 9000 (Orri and Darden, 2008). Nevertheless, no study has assessed the reliability of CON, ISO and ECC by using REV9000 (Technogym). In addition, no information concerning the reliability of lower limb strength imbalance indices using REV9000 are available and very few studies have examined the reliability of such when using Cybex (Sole et al., 2007) and Kin Com (Impellizzeri et al., 2008) in spite of their widespread use.

Thus, this study used the REV9000 isokinetic device (Technogym) on a group of healthy individuals and examined the reliability of the peak torque of the knee extensor and flexor muscles. In addition, it examined the reliability of commonly used strength imbalance indices, such as concentric hamstring (Hcon)/ concentric quadriceps (Qcon); eccentric quadriceps (Qecc)/ concentric quadriceps (Qcon); eccentric hamstring (Hecc)/ concentric hamstring (Hcon); eccentric hamstring (Hecc)/ concentric quadriceps (Qcon) and bilateral CON, ISO and ECC ratios. These assessment aims have never been studied with the use of a REV9000 dynamometer.

\section{Material and Methods}

\section{Procedures}

All sessions were carried out at the same time (4p.m.), approximately 3 hours after lunch. They were preceded by a 10 minute warm-up on an ergocycle (70rpm at $50 \mathrm{~W}$ ) and stretching exercises (for hamstrings and quadriceps) where subjects sat with their thighs at an angle of $85^{\underline{o}}$ to the trunk. The mechanical axis of the dynamometer was aligned with the lateral epicondyle of the knee. The trunk and thighs were stabilized with belts. The knee range of motion was $70^{\circ}$ (20 to $90^{\circ}$ of flexion); the lever arm was positioned at the distal third of the leg; torque was gravity-corrected and dynamometer calibration was performed before every session in accordance with the manufacturers' instructions. All sessions began with the assessment of the right lower limb and proceeded in the same order: $5 \mathrm{CON}$ contractions at $60^{\circ} \mathrm{s}^{-1}$ followed by ISO muscle contractions (flexion and extension) during 5 seconds (at $60^{\circ}$ of flexion) and 5 ECC contractions at $60^{\circ} \mathrm{s}^{-1}$.

A 90-second recovery period was allowed between CON, ISO and ECC tests. All subjects benefited from both visual feedback and verbal encouragement. The highest value for each testing condition was considered for statistical analysis. All procedures to assess muscular strength and power (Brown and Weir, 2001) were based on the recommendations of the American Society of Exercise Physiologists. All tests were performed with the use of the REV9000 (Technogym, Italy) isokinetic dynamometer and controlled by the same qualified technician.

The strength difference between the hamstrings and quadriceps of the same limb (unilateral) was calculated by the ratio between the peak torque produced concentrically during the isokinetic tests (Hcon:Qcon). The dynamic control ratio was calculated by the ratio between the peak torque produced eccentrically by the hamstrings (Hecc), and concentrically by the quadriceps (Qcon) (Impellizzeri et al., 2008). This variable (Hecc:Qcon) is an indicator of the extent in which hamstrings are capable of counteracting the anterior tibial shear induced by maximal quadriceps muscles (Aagaard et al., 1995). In fact, ECC antagonist/ CON agonist moment ratio has already been suggested to be a more valid indicator of muscular imbalance than the eccentric (ECC/ECC) or concentric (CON/CON) reciprocal parameters (Kellis and Baltzopoulos, 1995).

Previous research has already introduced different ways to calculate the bilateral lower limb strength asymmetry (Keays et al., 2003; Kuruganti and Seaman, 2006). The present study used a right/left comparison, this is, an absolute strength asymmetry of $40 \mathrm{Nm}$ in a subject with a stronger right leg (right leg $=200 \mathrm{Nm}$; left leg $=160 \mathrm{Nm}$ ) would correspond to 1.25 , however, if the same subject performed stronger with the left leg the ratio would be 0.80 (Impellizzeri et al., 2008).

\section{Analysis}

All strength imbalance indices and peak torque data were calculated with mean and 
standard deviation. Reliability concerned the degree in which individuals maintained their position in a sample involving repeated measurements (Atkinson and Nevill, 1998). This type of reliability was assessed with the intraclass correlation coefficient (ICC). ICC above 0.90 was considered high, between 0.80 and 0.90 as moderate and below 0.80 as low (Stuchlikova, 1995).

Detection of systematic biases was performed by using repeated ANOVA measurements while post-hoc differences were assessed by using the Bonferroni test (Atkinson and Nevill, 1998). Plotting the residual versus predicted values and calculating the Pearson's correlation coefficient with significant correlations indicated and examined heteroscedasticity, i.e. error depending on the magnitude of the mean.

Standard error of measurement (SEM) reflects the degree of an individuals' test score variation that might be expected from measurement error (Sleivert and Wenger, 1994) and in a case of a perfect agreement is equal to zero. This within-subject standard deviation is not only known as the typical error in a measurement but it also expresses the band of confidence around an individual's raw score. The most common way to calculate this statistic is by means of the following equation: $S E M=S D \times(1-I C C) 0.5$, where SD is the sample standard deviation and ICC is the calculated intraclass correlation coefficient (Atkinson and Nevill, 1998). The use of $\mathrm{SD}$ in the equation, in effect, partially "cancels out" the inter-individual variation that was used to calculate the ICC (Anthony, 1999). The comparison across the sample, measurement error was also expressed as the SEM\% [SEM\% = $(\mathrm{SEM} /$ mean $) \times 100$ ] to produce a unitless indicator of error magnitude.

The minimum detectable change (MDC) defined as $95 \%$ limits of agreement (Atkinson and Nevill, 1998; Weir, 2005), is also called the smallest real difference (SRD) and defined as 95\% confidence limit of the standard error of measurement (SEM). This threshold is required to detect statistically significant change in an individual when taking into account the variability associated with both the measurement technique and experimental sample (Beckerman et al., 2001; Beckerman et al., 1996). The SRD was calculated for each condition by using the equation $S R D$ or $M D C=1.96 \times 2^{0.5} \times S E M$.

The probability of type I error (alpha) was set at priori at 0.05 in all statistical analysis.

All procedures were performed with SPSS 17.0 statistical software (SPSS Inc., Chicago, IL, USA).

\section{Results}

Table 1 presents the mean \pm SD and ICC of the quadriceps peak torque. High reliability $(>0.90)$ was identified for the contraction mode. The highest and lowest ICC for peak torque was found in the eccentric contraction for the left knee flexor (0.97) and in ISO contraction of the left knee extensor (0.93), respectively. For concentric and eccentric contractions, the ICC was high in the interval of 0.95 to 0.97 (high reliability). Moreover, the ICC of ISO contractions had high reliability with values ranging between 0.93 and 0.96 . In what concerns the ICC of peak torque on the three attempts, all isokinetic and ISO strength outcomes showed high reliability for both extensor and flexor knee muscles. No significant differences were found between all sessions.

Table 2 presents the mean \pm SD and ICC of unilateral and bilateral strength imbalance ratios obtained in the three trials. ICC values for Hcon:Qcon and Hecc:Qecc were moderate-tohigh. Similarly, all ICC values for Hecc:Hcon and Qecc:Qcon showed moderate reliability. In regards to Hecc:Qcon ratio, the reliability was high for both right and left lower limbs. A significant effect of time was found for Qecc:Qcon ratio, which was lower in the first trial compared to the second and third trials. This effect was not found in the other unilateral ratios. The reliability of bilateral strength imbalance ratios obtained in the three trials was low-to-moderate. When compared to the bilateral hamstring ratio values (0.63-0.73), the ICC values for bilateral quadriceps ratios (0.71-0.81) were higher.

\section{Discussion}

The results of this study showed a high reliability of CON, ECC and ISO muscle strength assessment by using the REV9000 dynamometer for both knee extensor and flexor muscles. When comparing the results in the same session and between sessions, both reveal high reliability. However, ICC values found in the same session were always higher. 


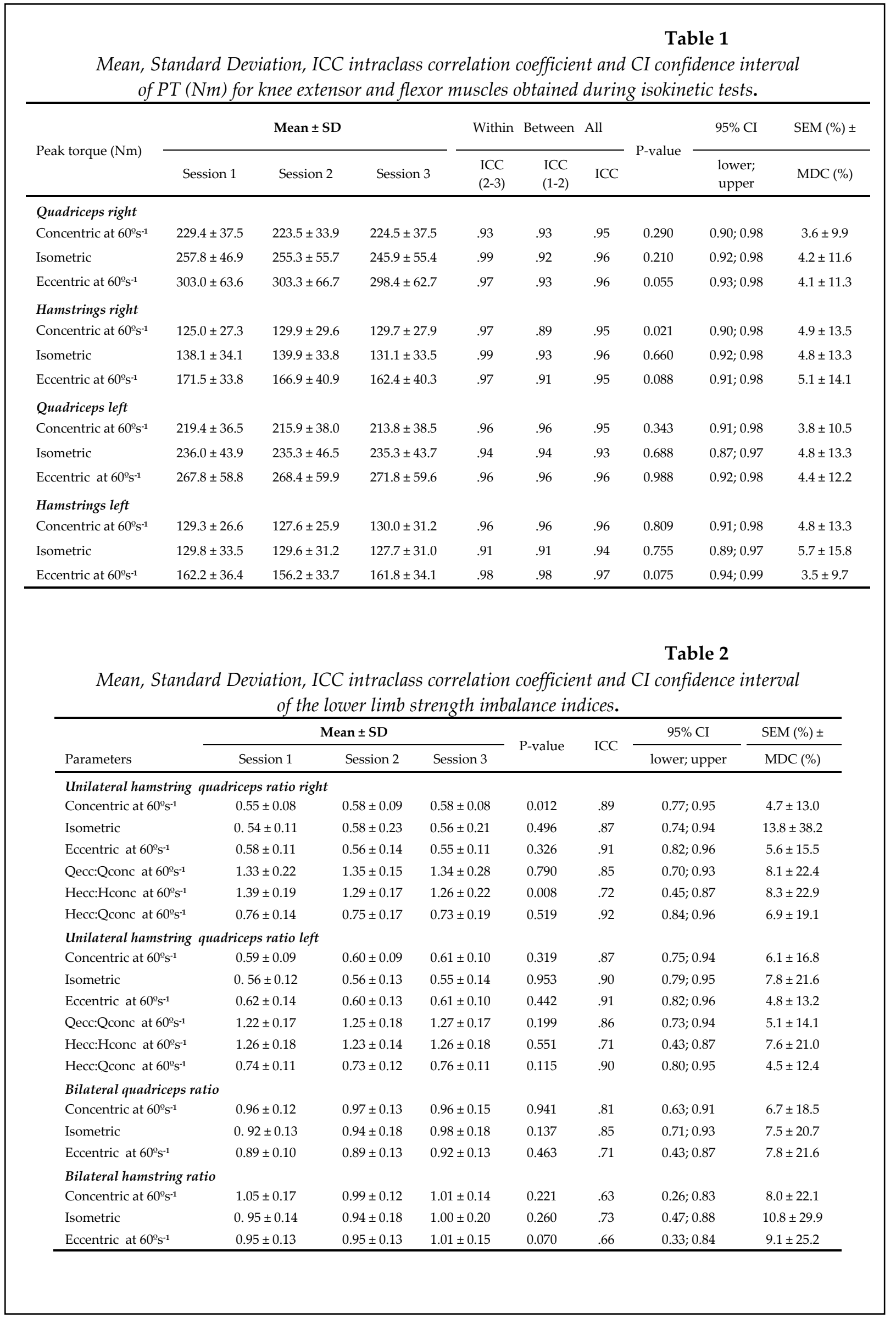




\section{Discussion}

When analysing the reliability among all sessions, the highest ICC for peak torque was found in the ECC contraction for the left knee flexor (0.97) and the lowest for the ICC in ISO contraction of the left knee extensor (0.93). For CON and ECC contractions, the ICC interval was 0.95 - 0.97 and 0.93 - 0.96 for ISO contractions.

These results were identical to a previous study (Maffiuletti et al., 2007) which showed values of 0.97 (Quad)/0.98(Ham) for concentric contraction at $60^{\circ} \mathrm{s}-1$ and 0.97 (Quad)/0.97(Ham) for ISO contractions. Another study (Impellizzeri et al., 2008) found similar ICC results of 0.95(Quad)/0.98(Ham) for the right lower limb and 0.95 (Quad)/0.93(Ham) for the left lower limb in concentric contractions at $60^{\circ} \mathrm{s}-1$. In what relates to eccentric contractions $\left(60^{\circ} \mathrm{s}-1\right)$, the same study had values of 0.96 (Quad)/0.94(Ham) for the right lower limb and 0.95(Quad)/0.97(Ham) for left lower limb.

The present study showed that the right knee flexor reliability peak torque was slightly higher than the knee extensor, which is not in accordance with previous findings (Impellizzeri et al., 2008; Li et al.,1996; Maffiuletti et al., 2007). Nevertheless, since the magnitude of the differences in ICC was very low, no parameter showed clear superior reliability when compared to the others.

The SEM percentual values of the absolute isokinetic strength measures ranged from $3.6 \%$ to $5.7 \%$ and the MDC from $9.7 \%$ to $15.8 \%$. These results were similar to a previous study that used a Biodex isokinetic device, which found SEM values of about $7 \%$ for knee extensors and $9 \%$ for knee flexors, with corresponding MDC ranging from $13 \%$ to $17 \%$ (Lund et al., 2005). SEM values ranging from $4 \%$ to $7 \%$ and MDC ranging from $10 \%$ to $20 \%$ with a Cybex 6000 Dauty \& Rochcongar (Dauty and Rochcongar, 2001) and SEM values ranging from $4.3 \%$ to $6.7 \%$ and MDC from $11.1 \%$ to $19 \%$ (Impellizzeri et al., 2008) have already been reported. Nevertheless, a previous study has found higher SEM and MDC values, however the assessed population was based on individuals with mild and moderate osteoarthritis of the knee and not on healthy subjects (Germanou et al., 2007).

The present study recognized the reliability of most common indices of strength imbalance ratios by using the REV9000. For bilateral quadriceps ratios, the ICC results were 0.81 for $\mathrm{CON}$ (at $60^{\circ} \mathrm{s}-1$ ), 0.85 for ISO and 0.71 for ECC contraction (at $60^{\circ} \mathrm{s}-1$ ). For bilateral hamstring ratios, ICC values were $0.63,0.73$ and 0.66 for CON, ISO and ECC contractions, respectively. These ICC values were slightly higher than those previously reported (Impellizzeri et al., 2008): 0.69 and 0.67, for the bilateral quadriceps ratio, using the $\mathrm{CON}$ and ECC peak torque at $60^{\circ} \mathrm{s}-1$. For bilateral hamstring ratios, the ICC values were 0.59 using the CON peak torque and 0.69 for ECC peak torque (at $\left.60^{\circ} \mathrm{s}-1\right)$. Another study showed even lower ICC values 0.42 at $30^{\circ} \mathrm{s}-1$ and 0.81 at $90^{\circ} \mathrm{s}-1$ for the bilateral quadriceps ratio (Hsu et al., 2002). However, one of the main reasons for these different results may be related to the participation of nine stroke patients.

Relatively to the Hcon:Qcon ratio, ICC values ranging from 0.36 to 0.93 in 10 healthy men after one session of familiarization had already been reported (Gleeson and Mercer, 1992). Another study found ICC values of 0.79 and 0.65 for the unilateral hamstring-to-quadriceps ratio using the CON peak torque (at $60^{\circ} \mathrm{s}-1$ ) of the right and left lower limbs (Impellizzeri et al., 2008). Moreover, ICC values of 0.43 at $60^{\circ}$ s- 1 were already found for the unilateral hamstring-toquadriceps ratio using the CON peak torque of the dominant lower limb (Sole et al., 2007). All these ICC results are considered low, however the present study found a moderate ICC of Hcon:Qcon ratio at $60^{\circ} \mathrm{s}-1$ (0.89 for the right leg and 0.87 for the left leg) and a high ICC for Hecc:Qecc ratio also at $60^{\circ} \mathrm{s}-1$ ( 0.91 for the right leg and 0.92 for the left leg). All together, these studies seem to show moderate reliability of these imbalance ratios, as previously suggested (Dauty et al., 2003; Gleeson and Mercer, 1992; Impellizzeri et al., 2008).

The present study provided higher ICC values for the Hecc:Qcon ratio measured at $60^{\circ} \mathrm{s}-1$ (0.92 for the right leg and 0.90 for the left leg) when compared to Hcon:Qcon ratios. These results are also higher than those found in previous reports. Moderate ICC values (0.87 for the right leg and 0.80 for the left leg) were already found for the Hecc:Qcon ratio measured at $60^{\circ} \mathrm{s}-1$ (Impellizzeri et al., 2008) and low ICC values (0.73 in dominant leg) were also reported for the 
Hecc/Qcon ratio at $60^{\circ}$ s-1 (Sole et al., 2007).

Subsequently, similar reliability values of knee extension and flexion measurements were found when comparing REV9000 with Cybex NORM and Con-Trex machines. Apparently, there were no differences in the results of reliability across all these devices; however there was a higher ICC with the REV9000 isokinetic dynamometer compared to the Cybex NORM and Con-Trex. In fact, it seems that reliability could present higher values if the subjects were more familiarized with the isokinetic tests because of the inherent learning effects.

In conclusion, the present study showed a high reproducibility of ECC, CON and ISO isokinetic strength for knee extensor and flexor muscles when using REV9000. As a consequence, it seems that this isokinetic device can be used for future sports performance studies and to improve the training process. The high reproducibility when using REV9000 may help coaches and clinicians to evaluate with accuracy the isometric and dynamic strength of the knee extensors and flexors, accelerate strength gains during rehabilitation processes and prevent possible risk factors of knee and hamstring injury or imbalances. A low-to-moderate reliability for the isokinetic strength bilateral ratios and a moderate reliability of Hcon:Qcon ratio was found. Additionally, high ICC for dynamic control ratio Hecc:Qcon and Hecc:Qecc ratio measured at $60^{\circ}$ s1 was found, indicating that these ratios can be used in rehabilitation and sports medicine.

\section{References}

Aagaard P, Simonsen EB, Trolle M, Bangsbo J, Klausen K. Isokinetic Hamstring Quadriceps Strength Ratio Influence from Joint Angular Velocity, Gravity Correction and Contraction Mode. Acta Physiologica Scandinavica, 1995; 154(4): 421-427

Anthony D. Understanding Advanced Statistics, a Guide for Nurses and Health Care Researchers: Churchill Livingstone; 1999

Atkinson G, Nevill AM. Statistical methods for assessing measurement error (reliability) in variables relevant to sports medicine. Sports Med, 1998; 26(4): 217-238

Bandy WD, Mclaughlin S. Intramachine and Intermachine Reliability for Selected Dynamic Muscle Performance Tests. J Orthop Sport Phys, 1993; 18(5): 609-613

Bardis C, Kalamara E, Loucaides G, Michaelides M, Tsaklis P. Intramachine and intermachine reproducibility of concentric performance: A study of the Con-Trex MJ and the Cybex Norm dynamometers. Isokinet Exerc Sci, 2004; 12(2): 91-97

Beckerman H, Roebroeck ME, Lankhorst GJ, Becher JG, Bezemer PD, Verbeek ALM. Smallest real difference, a link between reproducibility and responsiveness. Qual Life Res, 2001; 10(7): 571-578

Beckerman H, Vogelaar TW, Lankhorst GJ, Verbeek ALM. A criterion for stability of the motor function of the lower extremity in stroke patients using the Fugl-Meyer assessment scale. Scand J Rehabil Med, 1996; 28(1): 3-7

Blacker SD, Fallowfield JL, Bilzon JLJ, Willems MET. Within-day and between-days reproducibility of isokinetic parameters of knee, trunk and shoulder movements. Isokinet Exerc Sci, 2010; 18(1): 45-55

Brown LE, Weir JP. Accurate assessment of muscular strength and power. Journal of Exercise Physiology, 2001; 4(3): 1-21

Capranica L, Battenti M, Demarie S, Figura F. Reliability of isokinetic knee extension and flexion strength testing in elderly women. J Sport Med Phys Fit, 1998; 38(2): 169-176

Cotte T, Ferret JM. Comparative study of two isokinetics dynamometers: CYBEX NORM vs CON-TREX MJ. Isokinet Exerc Sci, 2003; 11(1): 37-43

Croisier JL. Factors associated with recurrent hamstring injuries. Sports Med, 2004; 34(10):681-695

Dauty M, Potiron-Josse M, Rochcongar P. Identification of previous hamstring muscle injury by isokinetic concentric and eccentric torque measurement in elite soccer player. Isokinet Exerc Sci, 2003; 11(3): 139144

Dauty M, Rochcongar P. Reproducibility of concentric and eccentric isokinetic strength of the knee flexors in elite volleyball players. Isokinet Exerc Sci, 2001; 9(2-3): 129-132 
Feiring D, Ellenbecker T, Dercheid G. Test-Retest reliability of the Biodex isokinetic dynamometer. J Orthop Sport Phys, 1990; 11(7): 298-300

Germanou E, Beneka A, Malliou P, Tsiganos G, Papalexandris S, Bikos C, Godolias G. Reproducibility of concentric isokinetic strength of the knee extensors and flexors in individuals with mild and moderate osteoarthritis of the knee. Isokinet Exerc Sci, 2007; 15(3): 151-164

Gleeson NP, Mercer TH. Reproducibility of Isokinetic Leg Strength and Endurance Characteristics of Adult Men and Women. Eur J Appl Physiol O, 1992; 65(3): 221-228

Hsu A, Tang P, Jan M. Test-retest reliability of isokinetic muscle strength of the lower extremities in patients with stroke. Arch Phys Med Rehab, 2002; 83(8): 1130-1137

Iga J, George K, Lees A, Reilly T. Reliability of assessing indices of isokinetic leg strength in pubertal soccer players. Pediatr Exerc Sci, 2006; 18(4): 436-445

Impellizzeri FM, Bizzini M, Rampinini E, Cereda F, Maffiuletti NA. Reliability of isokinetic strength imbalance ratios measured using the Cybex NORM dynamometer. Clin Physiol Funct I, 2008; 28(2): 113-119

Kannus P. Isokinetic Evaluation of Muscular Performance - Implications for Muscle Testing and Rehabilitation. Int J Sports Med, 1994; 15: S11-S18

Keays SL, Bullock-Saxton JE, Newcombe P, Keays AC. The relationship between knee strength and functional stability before and after anterior cruciate ligament reconstruction. J Orthopaed Res, 2003; 21(2): 231-237

Kellis E, Baltzopoulos V. Isokinetic Eccentric Exercise. Sports Med, 1995; 19(3): 202-222

Kuruganti U, Seaman K. The bilateral leg strength deficit is present in old, young and adolescent females during isokinetic knee extension and flexion. European journal of applied physiology, 2006; 97(3): $322-326$

Li RCT, Wu Y, Maffulli N, Chan KM, Chan JLC. Eccentric and concentric isokinetic knee flexion and extension: A reliability study using the Cybex 6000 dynamometer. Brit J Sport Med, 1996; 30(2): 156-160

Lund H, Sondergaard K, Zachariassen T, Christensen R, Bulow P, Henriksen M, Bliddal H. Learning effect of isokinetic measurements in healthy subjects, and reliability and comparability of Biodex and Lido dynamometers. Clin Physiol Funct I, 2005; 25(2): 75-82

Maffiuletti NA, Bizzini M, Desbrosses K, Babault N, Munzinger U. Reliability of knee extension and flexion measurements using the Con-Trex isokinetic dynamometer. Clin Physiol Funct I, 2007; 27(6): 346-353

Ordway NR, Hand N, Briggs G, Ploutz-Snyder LL. Reliability of knee and ankle strength measures in an older adult population. J Strength Cond Res, 2006; 20(1): 82-87

Orri JC, Darden GF. Technical report: Reliability and validity of the iSAM 9000 isokinetic dynamometer. J Strength Cond Res, 2008; 22(1): 310-317

Sleivert GG, Wenger HA. Reliability of Measuring Isometric and Isokinetic Peak Torque, Rate of Torque Development, Integrated Electromyography, and Tibial Nerve-Conduction Velocity. Arch Phys Med Rehab, 1994; 75(12): 1315-1321

Sole G, Hamren J, Milosavljevic S, Nicholson H, Sullivan SJ. Test-retest reliability of isokinetic knee extension and flexion. Arch Phys Med Rehab, 2007; 88(5): 626-631

Stuchlikova I. Statistics in kinesiology - Vincent,WJ. Cesk Psychol, 1995; 39(6): 568-569

Tredinnick TJ, Duncan PW. Reliability of Measurements of Concentric and Eccentric Isokinetic Loading. Phys Ther, 1988; 68(5): 656-659

Weir JP. Quantifying test-retest reliability using the intraclass correlation coefficient and the SEM. J Strength Cond Res, 2005; 19(1):231-240

\section{Corresponding author:}

\section{Alberto Carvalho}

Research Center in Sports Science, Health and Human Development (CIDESD), Portugal.

Adress: ISMAI - Instituto Superior da Maia. Av. Carlos Oliveira Campos - Castelo da Maia.

4475-690 Avioso S. Pedro

Phone: +351 229825319 ; Fax: +351229 825331

E-mail: acarvalho@ismai.pt 